\title{
BETWEEN ARTEMISININ AND DERIVATIVES WITH NEURAMINIDASE: A DOCKING STUDY INSIGHT
}

\author{
MOHAMMAD RIZKI FADHIL PRATAMA ${ }^{1 *}$, TUTUS GUSDINAR ${ }^{2}$
}

${ }^{1}$ Department of Pharmacy, Faculty of Health Sciences, Muhammadiyah University of Palangkaraya, Palangka Raya, Central Borneo, Indonesia. ${ }^{2}$ Department of Pharmacochemistry, School of Pharmacy, Bandung Institute of Technology, Bandung, West Java, Indonesia. Email: m.rizkifadhil@umpalangkaraya.ac.id

Objectives: This study aims to find the relationship between artemisinins and neuraminidase (NA) with molecular docking study and also to determine the most potent NA inhibitor from artemisinin and derivatives.

Methods: All ligands were sketched and optimized using Gaussian 03W with Hartree-Fock method basis sets 6-311G. Molecular docking was performed using AutoDock 4.2.3 toward NA in complexes with oseltamivir as co-crystal ligand. The main parameters used were the free energy of binding $(\Delta G)$ and dissociation constant $\left(\mathrm{K}_{\mathrm{i}}\right)$ as affinity marker.

Results: Artesunate provided most negative free $\Delta \mathrm{G}$ and lowest $\mathrm{K}_{\mathrm{i}}$ toward NA with $-9.55 \mathrm{kcal} / \mathrm{mol}$ and $100.66 \mathrm{nM}$, respectively. Artesunate shows higher affinity than oseltamivir with interactions between artesunate and amino acids at position 246 had important influences on artesunate affinity toward NA from H5N1.

Conclusion: In silico molecular docking results indicated that artesunate could be considered as NA inhibitor and should be potential to be developed as anti-influenza particularly to H5N1 with oseltamivir resistance.

Keywords: Artemisinin, Artesunate, Anti-influenza, H5N1, Neuraminidase.

(C) 2017 The Authors. Published by Innovare Academic Sciences Pvt Ltd. This is an open access article under the CC BY license (http://creativecommons. org/licenses/by/4. 0/) DOI: http://dx.doi.org/10.22159/ajpcr.2017.v10i8.18667

\section{INTRODUCTION}

Although avian influenza is no longer a frightening disease due to the development of H5N1 antivirus including oseltamivir and zanamivir, mutations of $\mathrm{H} 5 \mathrm{~N} 1$ could increase the resistance of influenza antiviral currently available [1]. Several H5N1 mutations even not only cause resistance to common neuraminidase inhibitor (NAI) but also cause transmission of avian influenza become airborne [2]. Sooner or later, avian influenza resistance toward known influenza antivirus will spread, and the need for new antiviral could be inevitable [3].

Compared with hemagglutinin, NA is a more ideal target for the development of anti-influenza compounds. Like oseltamivir and zanamivir, early developed NAIs considered to be effective for treatment and prophylaxis of avian influenza with minor side effects [4,5]. Several mutations in NA could reduce NAIs sensitivity [6]. However, several mutations only affect specific NAIs. For example, H274Y mutation caused resistance to oseltamivir (754-fold increase) and peramivir (260-fold increase), indicated that NA was still had potency as antiinfluenza targets therapy [7].

Discovery of NAIs with oseltamivir-based compounds resulting in the development of several known NAIs with more effectiveness example like laninamivir, a long action NAI [8]. However, since almost every known NAIs share similar molecular structure, the potency of crossresistances between NAIs is still high $[9,10]$. Discovery of secondary metabolites from medicinal plants with antiviral especially antiinfluenza activity is a key to obtain new NAI with high effectiveness. Some herbs extract shows anti-influenza activity and appear to be safe for human consumption [11]. One of the traditional medicinal plants with potent antiviral activity was Artemisia annua or qinghaosu. Artemisinins, its main secondary metabolites was known for having several antiviral activities $[12,13]$. However, to date still, no researches linking between artemisinin and derivatives with anti-influenza properties toward NA inhibition. In this study, we conducted in silico study to determine the most potent artemisinin derivatives as NAI with molecular docking method. We have selected seven artemisinin derivatives as test ligands that already used as antimalarial therapy or still in preclinical phase. Our purpose is to obtain information about other therapeutics activity that could be developed as drug repurposing from these compound class.

\section{METHODS}

\section{Preparation of ligands and receptor}

Structures of artemether, artemisinin, artemisone, artemotil, artelinic acid, artesunate, and dihydroartemisinin were sketched using GaussView 3.08 Software from Gaussian, Inc. All structures were geometry optimized by Hartree-Fock method basis sets 6-311G with Gaussian 03 W Software from Gaussian, Inc. Geometry optimization provided the most ideal conformation of following compounds that approaching conformation of these compounds in nature [14]. Optimized structures format changed from .log to .pdb using Open Babel 2.3.2 Software [15]. Docking program used in this study was AutoDock 4.2.3 from the Scripps Research Institute [16]. The molecular structure of NA H5N1 in complexes with oseltamivir (protein data bank [PDB] ID 2HU4) was obtained from website of PDB www.rscb.org with oseltamivir binding site chosen as active site since this site already known for the development of NAI [17].

\section{Validation of docking process}

The method used for molecular docking validation was pose selection using co-crystal structure by redocking it into active site of NA protein. Thus, redocking was performed with oseltamivir on NA active site. The parameters observed in validation is root-mean-square deviation (RMSD) of each ligand co-crystal at selected binding site [18]. Docking 
programs are preferred to predict results from experimental poses with RMSD no more than $2 \AA$. Smaller RMSD indicate that position of redocking ligand was closer to crystallography ligand $[19,20]$.

\section{Docking studies}

Molecular docking for all test ligand performed in same way as validation process using with similar grid box size and position [21]. The main parameter used in docking process were free energy of binding $(\Delta \mathrm{G})$, dissociation constant $\left(\mathrm{K}_{\mathrm{i}}\right)$, amino acids residues, and number of hydrogen bonds. Ligand affinity to receptor in docking method was determined by $\Delta \mathrm{G}$ and $\mathrm{K}$ scores. More negative $\Delta \mathrm{G}$ and lower $\mathrm{K}_{\mathrm{i}}$ indicated higher ligand affinity toward active site of used receptor [22-24]. Test ligand with the highest affinity was compared with validation result of co-crystal ligand of active site to determine the potency of test ligand as NAI [14].

\section{RESULTS AND DISCUSSION}

Docking validation was done with redocking method using AutoDock 4.2.3. Validation was performed on the entire binding site using co-crystal ligand of selected receptor. Using of entire binding site was purposed to identify any other potential active site at NA receptor. However, redocking result showed that oseltamivir as cocrystal ligand docked into similar position like crystallography result [14].

Redocking results from this study were provided RMSD value wand almost at stacked position with crystallography results (Fig. 1), indicated that receptor $2 \mathrm{HU} 4$ was valid for molecular docking purpose [20]. Other parameters observed in validation was $\Delta G, K_{i,}$ amino acids residues, and number of hydrogen bonds of cocrystal ligand as shown in Table 1.

Test ligands were sketched and performed geometry optimization. Hartree-Fock method was used with basis set 6-311G for geometry optimization (Table 2). This method was ab initio approximation with relatively high confidence rate for in silico analysis [14].

Docking was performed using AutoDock 4.2.3 at active site of NA receptor with 100 genetic algorithms runs to improve accuracy of docking result [16]. For each test ligand, one poses with most negative $\Delta G$ and lowest $K$ was selected as representatives of test ligand [14]. The docking results data of seven ligands to NA were compared each other as shown in Table 3. Compared to other ligands, artesunate had the most negative $\Delta G$ and lowest $K_{i}$ thus had the highest affinity toward NA than other artemisinin derivatives. All ligands also provided negative score of $\Delta \mathrm{G}$, indicated that interaction between NA receptor active site and ligands will occur spontaneously [19].

Comparison of amino acids residues and number of hydrogen bonds between docking results of oseltamivir as co-crystal ligand and artesunate as test ligand then performed to analyzed similarities and differences between docking results of two compounds. Comparison results shown in Table 4 indicated slight differences in amino acids residues and number of hydrogen bonds between oseltamivir and artesunate docking results. Interestingly, artesunate had slight higher affinity than oseltamivir.

Whether artesunate had the same activity with oseltamivir or not was still unclear. However, since most amino acids residues which interacted with oseltamivir also interacted with artesunate ( 8 out of 10 ), there was possible that artesunate also inhibits NA like oseltamivir [25]. Since NA inhibition was one of primary target for influenza therapy, this study results indicated that artesunate had potency as NAI.

There is only one amino acid residue that forms in docking result of artesunate in position 246, which do not occurs in other ligands docking result. As comparison, docking result of artemisone had many similarity with docking result of artesunate, the differences occurs only in 3 out of 13 amino acids residues despite artesunate gave much number of hydrogen bonds than artemisone. However, compared to artesunate, artemisone gave lower affinity toward NA receptor active site (Table 3). This result proved that presence interaction at amino acid residue in position 246 had important influence on artesunate affinity toward NA receptor.

More observation conducted to reveal interaction between artesunate and NA receptor active site as shown in Fig. 2. Artesunate was docked into cleavage between set of $\beta$-strand peptides (yellow in left Fig. 2) in $\mathrm{N}$-terminal lobes of tyrosine kinase domain. On the other hand, interactions between artesunate and amino acids residues dominated with hydrophilic interactions with five hydrogen bonds formed (right Fig. 2), even more than oseltamivir (Table 4). We also compared position of artesunate with oseltamivir directly which obtained from

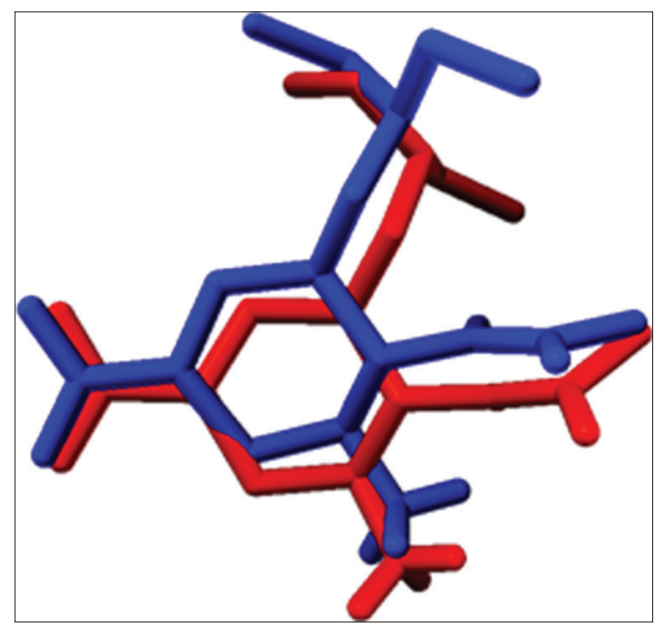

Fig. 1: Results of validation from oseltamivir and neuraminidase; root-mean-square deviation=1.183 $\AA$ (Red: Oseltamivir redocking result; Blue: Oseltamivir crystallography result)

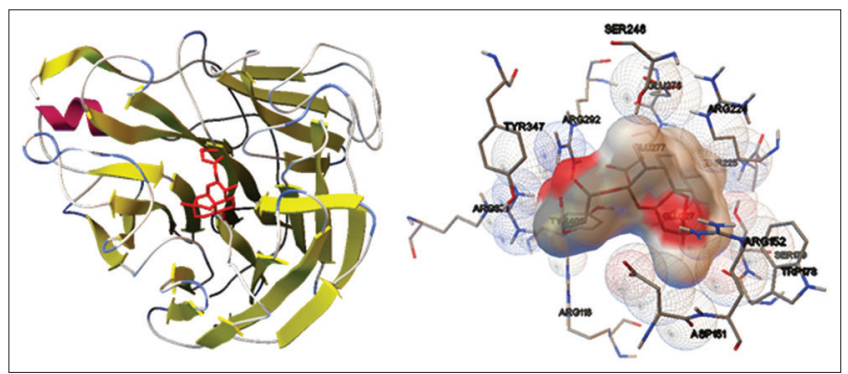

Fig. 2: Docking results of artesunate and neuraminidase (NA)

(Left: Artesunate position in NA; Right: Interactions between artesunate and amino acids residues of NA receptor active site)

Table 1: Validation results of NA receptors PDB ID 2HU4 with co-crystal ligand oseltamivir

\begin{tabular}{lllllll}
\hline Receptor & Ligand & RMSD $(\AA)$ & $\Delta \mathbf{G}(\mathbf{k c a l} / \mathbf{m o l})$ & $\mathbf{K}_{\mathrm{i}}(\boldsymbol{\mu M})$ & Amino acids residues & $\begin{array}{l}\text { Number of } \\
\text { hydrogen bonds }\end{array}$ \\
\hline NA & Oseltamivir & 1.183 & -9.26 & 0.16253 & $\begin{array}{l}\text { 118-Arg, 119-Glu, 151-Asp, 152-Arg, 178-Trp, 224-Arg, } \\
\text { 277-Glu, 292-Arg, 371-Arg, 406-Tyr }\end{array}$ & 4 \\
\hline
\end{tabular}

NA: Neuraminidase, PDB: Protein data bank, RMSD: Root-mean-square deviation, $\Delta \mathrm{G}$ : Energy of binding, K: Dissociation constant 
Table 2: 2D and 3D structure of ligands

Compounds

Artemisinin (ART)

Artemether (ARM)

Artemisone (ARO)

Artemotil (ARL)

Artelinic acid (ARA)

Artesunate (ARS)

Dihydroartemisinin (DHA)

\section{D Structure}
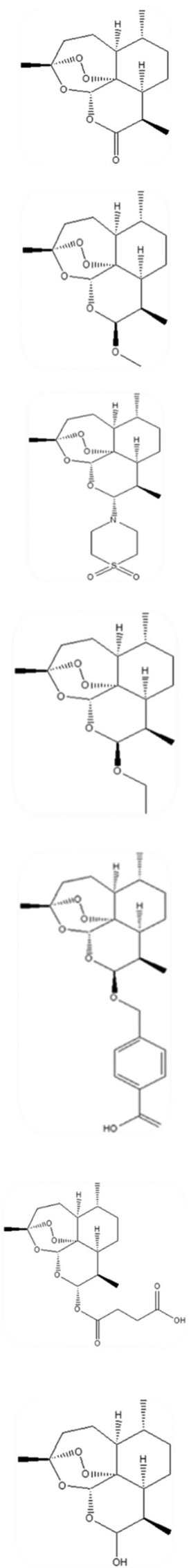

3D Structure
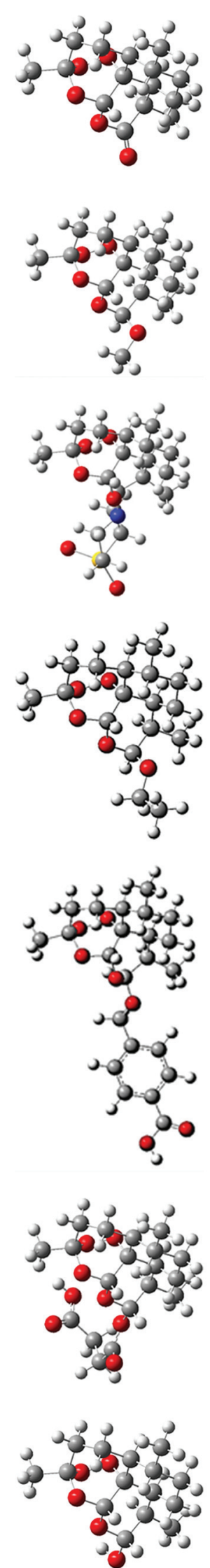
Table 3: Docking results of artemisinin derivatives at NA receptor

\begin{tabular}{|c|c|c|c|c|c|c|c|}
\hline Parameters & ART & ARM & ARO & ARL & ARA & ARS & DHA \\
\hline$\Delta \mathrm{G}(\mathrm{kcal} / \mathrm{mol})$ & -6.85 & -6.10 & -7.84 & -6.31 & -7.82 & -9.55 & -6.98 \\
\hline $\mathrm{K}_{\mathrm{i}}(\mu \mathrm{M})$ & 9.56 & 33.54 & 1.78 & 23.51 & 1.87 & 0.10066 & 7.65 \\
\hline \multirow[t]{17}{*}{ Amino acids residues } & - & - & 118-Arg & - & 118-Arg & 118-Arg & - \\
\hline & - & - & - & - & - & - & - \\
\hline & - & - & - & - & - & - & - \\
\hline & - & 151-Asp & 151-Asp & 151-Asp & - & 151-Asp & 151-Asp \\
\hline & 152-Arg & 152-Arg & - & 152-Arg & 152-Arg & 152-Arg & 152-Arg \\
\hline & 178-Trp & 178-Trp & 178-Trp & 178-Trp & - & 178-Trp & 178-Trp \\
\hline & 179-Ser & 179-Ser & 179-Ser & 179-Ser & 179-Ser & 179-Ser & - \\
\hline & - & - & - & - & 222-Ile & - & - \\
\hline & 224-Arg & 224-Arg & 224-Arg & 224-Arg & - & 224-Arg & 224-Arg \\
\hline & 225-Thr & 225-Thr & 225-Thr & 225-Thr & - & 225-Thr & - \\
\hline & 227-Glu & 227-Glu & 227-Glu & 227-Glu & - & 227-Glu & - \\
\hline & - & - & - & - & - & 246-Ser & - \\
\hline & 276-Glu & - & 276-Glu & - & - & 276-Glu & - \\
\hline & - & - & - & - & - & - & - \\
\hline & - & - & 347-Tyr & - & 347-Tyr & 347-Tyr & - \\
\hline & - & - & 371-Arg & 371-Arg & 371-Arg & 371-Arg & - \\
\hline & - & - & 406-Tyr & 406-Tyr & 406-Tyr & 406-Tyr & - \\
\hline Number of hydrogen bonds & 1 & 1 & 1 & 1 & 3 & 5 & 3 \\
\hline
\end{tabular}

ART: Artemisinin, ARM: Artemether, ARO: Artemisone, ARL: Artemotil, ARA: Artelinic acid, ARS: Artesunate, DHA: Dihydroartemisinin, NA: Neuraminidase, $\triangle$ G: Energy of binding, $\mathrm{K}_{\mathrm{i}}$ : Dissociation constant

Table 4: Comparison of docking results between oseltamivir and artesunate

\begin{tabular}{lll}
\hline Parameters & Oseltamivir & Artesunate \\
\hline$\Delta \mathrm{G}(\mathrm{kcal} / \mathrm{mol})$ & -9.26 & -9.55 \\
$\mathrm{~K}_{\mathrm{i}}(\mathrm{nM})$ & 162.53 & 100.66 \\
Amino acids residues & $118-\mathrm{Arg}$ & $118-\mathrm{Arg}$ \\
& $119-\mathrm{Glu}$ & - \\
& - & - \\
$151-\mathrm{Asp}$ & $151-\mathrm{Asp}$ \\
& $152-\mathrm{Arg}$ & $152-\mathrm{Arg}$ \\
& - & - \\
& $178-\mathrm{Trp}$ & $178-\mathrm{Trp}$ \\
& - & $179-\mathrm{Ser}$ \\
& - & - \\
& $224-\mathrm{Arg}$ & $224-\mathrm{Arg}$ \\
& - & $225-\mathrm{Thr}$ \\
& - & $227-\mathrm{Glu}$ \\
& - & $246-\mathrm{Ser}$ \\
& - & $276-\mathrm{Glu}$ \\
& $277-\mathrm{Glu}$ & $277-\mathrm{Glu}$ \\
& $292-\mathrm{Arg}$ & - \\
\hline
\end{tabular}

$\Delta \mathrm{G}$ : Energy of binding, $\mathrm{K}$ : Dissociation constant

redocking method. As result, we overlay both docking results structure as shown in Fig. 3.

Both artesunate and oseltamivir docked into similar position at the active site of NA receptor active site. However, endoperoxide chain of artesunate had different behavior with side chain of oseltamivir. Aside from other artemisinins, interactions of artesunate and NA were dominated by interactions at amino acid's in position 224-277 (Table 4). Total six interactions were formed in artesunate compared with two in oseltamivir. This results could lead in conclusion that artesunate had higher affinity toward NA than oseltamivir caused by more interactions occurred at those position. Hence, interactions at amino acids residues number 224-277 could have important

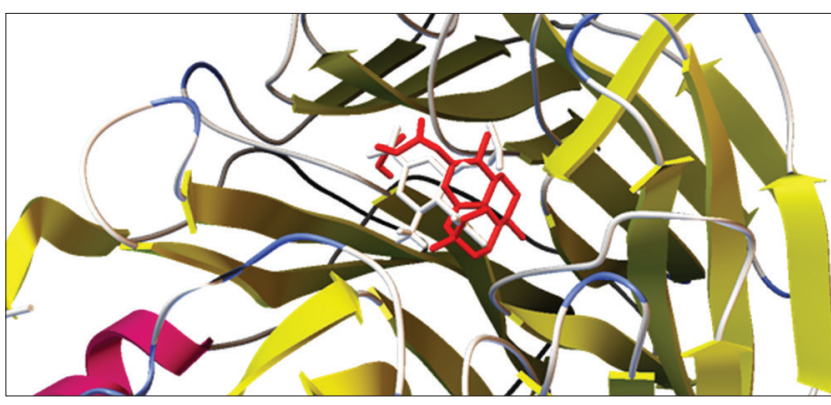

Fig. 3: Comparison between docking results of artesunate and oseltamivir (Red: Artesunate; White: Oseltamivir)

influences toward activity as NAI, primarily amino acid serine at position 246 .

\section{CONCLUSION}

This study was successfully described linking between artemisinin and derivatives with NA, even gave interesting result where artesunate had higher affinity than oseltamivir at NA active site. Artesunate provided $\Delta \mathrm{G}$ and $\mathrm{K}_{\mathrm{i}}-9.55 \mathrm{kcal} / \mathrm{mol}$ and $100.66 \mathrm{nM}$, respectively. These result open up opportunities to develop artesunate as potent anti-influenza especially one with oseltamivir resistance. More researches should be done to optimize the interactions mainly in amino acid position 224-277, especially with 246-serine. Designing novel NAI derives from artesunate should be focus at those amino acids residues. Thus, this study clearly indicates a promising potential of artemisinin and derivatives to be develop as NAI for anti-influenza therapy.

\section{REFERENCES}

1. Smith JR. Oseltamivir in human avian influenza infection. J Antimicrob Chemother 2010;65 Suppl 2:25-33.

2. Maurer-Stroh S, Li Y, Bastien N, Gunalan V, Lee RT, Eisenhaber F, et al. Potential human adaptation mutation of influenza A(H5N1) virus, Canada. Emerg Infect Dis 2014;20(9):1580-2.

3. Aiki-Raji CO, Aguilar PV, Kwon YK, Goetz S, Suarez DL, Jethra AI, et al. Phylogenetics and pathogenesis of early avian influenza viruses (H5N1), Nigeria. Emerg Infect Dis 2008;14(11):1753-5. 
4. Eyer L, Hruska K. Antiviral agents targeting the influenza virus: A review and publication analysis. Vet Med 2013;58(3):113-85.

5. Moscona A. Neuraminidase inhibitors for influenza. N Engl J Med 2005;353(13):1363-73

6. McKimm-Breschkin JL. Influenza neuraminidase inhibitors: Antiviral action and mechanisms of resistance. Influenza Other Respir Viruses 2012;7 Suppl 1:25-36

7. Abed Y, Baz M, Boivin G. Impact of neuraminidase mutations conferring influenza resistance to neuraminidase inhibitors in the N1 and N2 genetic backgrounds. Antivir Ther 2006;11(8):971-6.

8. von Itzstein $M$. The war against influenza: Discovery and development of sialidase inhibitors. Nat Rev Drug Discov 2007;6(12):967-74.

9. Nguyen HT, Fry AM, Gubareva LV. Review: Neuraminidase inhibitor resistance in influenza viruses and laboratory testing methods. Antiviral Ther 2012;17:159-73.

10. Nitsch-Osuch A, Brydak LB. Influenza viruses resistant to neuraminidase inhibitors. Acta Biochim Pol 2014;61(3):505-8.

11. Hudson JB. Review: The use of herbal extracts in the control of influenza. J Med Plant Res 2009;3(13):1189-95.

12. Efferth T, Romero MR, Wolf DG, Stamminger T, Marin JJ, Marschall $\mathrm{M}$. The antiviral activities of artemisinin and artesunate. Clin Infect Dis 2008;47(6):804-11.

13. Karamoddini MK, Emami SA, Ghannad MS, Sani EA, Sahebkar A. Antiviral activities of aerial subsets of Artemisia species against herpes simplex virus Type 1 (HSV1) in vitro. Asian Biomed 2011;5(1):63-8.

14. Cosconati S, Forli S, Perryman AL, Harris R, Goodsell DS, Olson AJ. Virtual screening with autodock: Theory and practice. Expert Opin Drug Discov 2010;5(6):597-607.

15. O’Boyle NM, Banck M, James CA, Morley C, Vandermeersch T, Hutchison GR. Open Babel: An open chemical toolbox. J Cheminform 2011;3:33.
16. Morris GM, Huey R, Lindstrom W, Sanner MF, Belew RK, Goodsell DS, et al. AutoDock4 and AutoDockTools4: Automated docking with selective receptor flexibility. J Comput Chem 2009;30(16):2785-91

17. Russell RJ, Haire LF, Stevens DJ, Collins PJ, Lin YP, Blackburn GM, et al. The structure of $\mathrm{H} 5 \mathrm{~N} 1$ avian influenza neuraminidase suggests new opportunities for drug design. Nature 2006;443(7107):45-9.

18. Miladiyah I, Jumina J, Haryana SM, Mustofa M. In Silico molecular docking of xanthone derivatives as cyclooxygenase-2 inhibitor agents. Int J Pharm Pharm Sci 2017;9(3):98-104

19. Kontoyianni M, McClellan LM, Sokol GS. Evaluation of docking performance: Comparative data on docking algorithms. J Med Chem 2004;47(3):558-65.

20. Bissantz C, Folkers G, Rognan D. Protein-based virtual screening of chemical databases 1. Evaluation of different docking/scoring combinations. J Med Chem 2000;43(25):4759-67.

21. Umashankar V, Gurunathan S. Drug discovery: An appraisal. Int J Pharm Pharm Sci 2015;7(4):59-66.

22. Kim R, Skolnick J. Assessment of programs for ligand binding affinity prediction. J Comput Chem 2008;29(8):1316-31.

23. Munusami P, Indu AG, Vasavi CS, Diyva G. Molecular docking studies on flavonoid compound: An insight into aromatase inhibitors. Int $\mathrm{J}$ Pharm Pharm Sci 2014;6(10):141-8.

24. Thangathirupathi A, Naushad A, Natarajan P, Ramesh KD. Molecular docking studies of andrographolide with xanthine oxidase. Asian J Pharm Clin Res 2013;6(2):300-2.

25. Okomo-Adhiambo M, Sleeman K, Ballenger K, Nguyen HT, Mishin VP, Sheu TG, et al. Neuraminidase inhibitor susceptibility testing in human influenza viruses: A laboratory surveillance perspective. Viruses 2010;2(10):2269-89. 